\title{
Can personalized medicine mitigate confirmation bias in mental health?
}

\author{
Giampaolo Perna, ${ }^{1,2}$ (ID Charles B. Nemeroff ${ }^{3}$ iD \\ ${ }^{1}$ Department of Biological Sciences, Humanitas University, Pieve Emanuele, Milan, Italy. ${ }^{2}$ Department of Clinical Neurosciences, Villa San \\ Benedetto Menni, Hermanas Hospitalarias, Albese con Cassano, Como, Italy. ${ }^{3}$ Department of Psychiatry and Behavioral Sciences, Dell \\ Medical School, University of Texas at Austin, Austin, TX, USA.
}

In the 20th century, the basis of both medical and psychiatric practice progressed from clinical judgment acquired over the physician's career to evidence-based medicine, which is informed by studies or meta-analyses from which general recommendations based on an "average result" are derived. Concomitant with this shift, clinical practice guidelines for medical diseases and specific psychiatric disorders began appearing, which recommend systematic clinical and therapeutic options developed by expert committees or consensus conferences based on the strength of the available evidence.

Health systems have invested considerable resources implementing clinical practice guidelines, believing that they will reduce variation in care, minimize medical errors, and make care more evidence based. Beginning in 1993, the UK created two organizations to develop national evidence-based guidelines, the National Institute for Clinical Excellence and the Scottish Intercollegiate Guidelines Network.

Unfortunately, studies assessing compliance with clinical practice guidelines have reported several examples of disorders that are worsened by inadequate guideline implementation, such as sepsis and asthma. Moreover, it has been determined that evidence-based practices take an average of 17 years for routine incorporation in healthcare institutions. Approximately half of the guidelines never reach widespread clinical usage. ${ }^{1}$

In psychiatry, due to the poorly developed ${ }^{2}$ pathway from evidence-based guidelines to evidence-based practice, it remains unclear whether treatment guidelines improve patient outcomes and mental health services. ${ }^{2}$ This is not surprising, due to the intrinsic complexity and heterogeny of psychiatric disorders and the lack of laboratory or imaging tests to validate the diagnosis.

Due to these issues, in the past 10 years there has been a dramatic growth in implementation science, which focuses on promoting research and translating evidencebased medicine to routine practice. Regardless of whether filling the evidence-to-practice gap has been the traditional concern of healthcare researchers, substantial investment in implementation science has recently been made by federal funding agencies in the USA. ${ }^{3}$

Correspondence: Giampaolo Perna, Humanitas University, via Rita Levi Montalcini 4, 20090, Pieve Emanuele, MI, Italy.

E-mail: giampaolo.perna@ @unimed.eu

Submitted Jul 20 2021, accepted Jul 20 2021, Epub Oct 152021.
Implementation intervention aims to address identified barriers to putting evidence-based practices into routine clinical usage and changing behavior on the patient, clinician, system, and policy levels. Although several causes of implementation failure have been identified, such as the characteristics of clinical practice guidelines, the continuing education of physicians and other health professionals represents a strategic step toward improved care processes and clinical outcomes. However, passive educational approaches, such as guideline dissemination, are generally ineffective for changing behavior. Conversely, the evidence has supported methods that engage clinicians in collaborative problem-solving, facilitating peer norm enforcement and practice-based change, such as educational outreach visits to health professionals in their work settings, auditing, and feedback (confidentially comparing clinician performance with that of a peer group).

Certain cognitive factors should be considered in implementation failure assessment. When making judgments or decisions, people often rely on simplified information-processing strategies, which can lead to systematic errors (cognitive biases), the most intriguing of which is confirmation bias (CB). Blumenthal-Barby \& Krieger's ${ }^{4}$ systematic review supported the relevance of cognitive biases in decision-making by both patients and clinicians. They found that $80 \%$ of studies involving medical personnel and $61 \%$ of those involving patients had cognitive and heuristic biases regarding medical decision-making. Accordingly, all 20 studies included in Saposnik's ${ }^{5}$ systematic review found at least one cognitive bias or personality trait that affected the medical decision-making of physicians and had repercussions on diagnostic accuracy and patient management.

For these reasons, we want to focus here on the psychological construct of $\mathrm{CB}$, an important determinant of human thought and behavior that is a potential factor in the failure to implement evidence-based practice.

$\mathrm{CB}$ is the tendency to search for, interpret, and recall information in a way that confirms or supports one's prior beliefs or values. As proposed by some authors, defending one's view after taking a position on an issue is a 
primary human need. It follows that even if the belief is based on an impartial evaluation of the evidence, CB can still manifest itself subsequently.

In practice, people defend their beliefs by seeking information that supports their preexisting views or by interpreting the information congruently with their initial position. Conversely, we tend to avoid information that can challenge our preexisting beliefs or support alternative possibilities. It is assumed that the purpose of selective exposure to information is to avoid an unpleasant state, known as cognitive dissonance, which arises from dissonant cognitions or cognitive conflicts (defense motivation). Interestingly, $\mathrm{CB}$ is affected by the quality of information available during the selection process: regardless of whether the information supports or refutes the initial belief, high-quality information tends to reinforce $\mathrm{CB}$, while low-quality information tends to decrease it.

Most debiasing techniques against $\mathrm{CB}$ are designed to shift cognitive processing from an automatic, heuristic mode of thinking to a controlled, rule-governed mode, aiming to "override" the automatic propensity to consider only one's point of view. ${ }^{6}$ Unfortunately, despite the broad array of interventions that have been proposed to reduce cognitive error in medicine, including computer-based decision support systems or information restructuring, evidence for their effectiveness in healthcare remains limited. ${ }^{7}$ In particular, there is no strong evidence that cognitive error is reduced by calling attention to errorprone clinical situations or by using tools to mitigate bias.

We believe that personalized medicine, which has led to considerable improvement in treatment outcomes in oncology and infectious diseases, may help overcome the research-to-practice gap regarding the impact of $\mathrm{CB}$ on physician decisions. In recent decades, the personalized medicine approach has been widely studied in the treatment of mental disorders, leading to the development of personalized medicine in psychiatry (PMP).

PMP proposes to establish clinical decisions for disease prevention and treatment that consider individual variability in clinical, biological, environmental, and lifestyle factors, tailoring the interventions to each patient's unique characteristics. ${ }^{8}$

The predictive models of diagnosis and outcomes in PMP are based on the collection of very large-scale data (big data), which are analyzed with advanced computational tools, such as "supervised" machine learning that develops algorithms, providing the best possible prediction when applied to a single patient, i.e., predicting a response before a therapy is administered. ${ }^{9}$

Recent technological advances, such as electronic medical records, which contain a wealth of real-world patient information collected during standard clinical care, and electronic data capture tools (smartphones, activity monitors, wearable devices, etc.), which monitor behavior and physiological changes, have facilitated big data collection. PMP has also benefitted from additional data from omics sciences (genomic, epigenetic, transcriptomic, proteomic, metabolomic assessments, etc.) and imaging protocols. In addition to these, however, deep phenotyping is an absolute requirement. By this we mean a detailed scientifically based series of assessments that characterize the patient's symptoms, including measures of disease severity. None of the abovementioned techniques can yield fruitful results unless the latter is performed. We have learned this lesson from large genome-wide association studies that, for example, only utilize electronic medical records.

Of note, clinical practice guideline-based medical practice requires adherence to a hierarchy of evidence-based choices suggested by experts that do not necessarily coincide with the clinician's view/experience, which can lead to $C B$ as a defensive reaction. Conversely, in the PMP approach, machine learning analysis of a vast amount of objective data (without a priori judgments of their value from a diagnostic or therapeutic point of view) could protect clinicians from CB. Along with its technical methods, the atheoretical basis of PMP could help overcome $\mathrm{CB}$ in physician decisions.

Along with technological innovation, the successful transitioning of validated PMP platforms into clinical practice will require a series of advances. In addition to the challenges associated with healthcare economics, ethics, and data privacy, the impact of PMP and clinical practice guideline-based medicine on clinician decisionmaking and patient outcomes should be addressed.

\section{Acknowledgements}

CBN has received research grants from the National Institutes of Health (NIH); has served in the scientific advisory boards of the Brain \& Behavior Research Foundation (BBRF), Anxiety and Depression Association of America (ADAA), Skyland Trail, and the Laureate Institute for Brain Research (LIBR); and has served in the board of directors of Gratitude America and ADAA.

\section{Disclosure}

GP has received consulting fees from Medibio, Menarini, Lundbeck, and Pfizer. CBN has received consulting fees from ANeuroTech (division of Anima BV), Signant Health, Sunovion Pharmaceuticals, Janssen Research \& Development LLC, Magstim, Navitor Pharmaceuticals, Intra-Cellular Therapies, EMA Wellness, Acadia Pharmaceuticals, Axsome, Sage, BioXcel Therapeutics, Silo Pharma, XW Pharma, Neuritek, Engrail Therapeutics, and Corcept Therapeutics Pharmaceuticals Company; is a stockholder in Xhale, Seattle Genetics, Antares, BI Gen Holdings, Corcept Therapeutics Pharmaceuticals Company, EMA Wellness, and TRUUST Neuroimaging; has served in the scientific advisory boards of ANeuroTech (division of Anima BV), Signant Health, Magnolia CNS, Heading Health, and TRUUST Neuroimaging; has served in the board of directors of Xhale Smart; and is coauthor of two patents (Method and devices for transdermal delivery of lithium [US 6,375,990B1], and Method of assessing antidepressant drug therapy via transport inhibition of monoamine neurotransmitters by ex vivo assay [US 7,148,027B2]). 


\section{References}

1 Bauer MS, Kirchner JA. Implementation science: what is it and why should I care? Psychiatry Res. 2020;283:112376.

2 Bighelli I, Ostuzzi G, Girlanda F, Cipriani A, Becker T, Koesters M, et al. Implementation of treatment guidelines for specialist mental health care. Cochrane Database Syst Rev. 2016;12:CD009780.

3 Bowser DM, Henry BF, McCollister KE. Cost analysis in implementation studies of evidence-based practices for mental health and substance use disorders: a systematic review. Implement Sci. 2021;16:26.

4 Blumenthal-Barby JS, Krieger $\mathrm{H}$. Cognitive biases and heuristics in medical decision making: a critical review using a systematic search strategy. Med Decis Making. 2015;35:539-57.
5 Saposnik G, Redelmeier D, Ruff CC, Tobler PN. Cognitive biases associated with medical decisions: a systematic review. BMC Med Inform Decis Mak. 2016;16:138.

6 Ludolph R, Schulz PJ. Debiasing health-related judgments and decision making: a systematic review. Med Decis Making. 2018;38: 3-13.

7 O'Sullivan ED, Schofield SJ. A cognitive forcing tool to mitigate cognitive bias - a randomised control trial. BMC Med Educ. 2019;19:12.

8 Perna G, Nemeroff CB. Personalized medicine in psychiatry: back to the future. Pers Med Psychiatry. 2017;1:1-2.

9 Perna G, Grassi M, Caldirola D, Nemeroff CB. The revolution of personalized psychiatry: will technology make it happen sooner? Psychol Med. 2018;48:705-13. 